Research Article: New Research / Disorders of the Nervous System

\title{
Differential electrographic signatures generated by mechanistically-diverse seizurogenic compounds in the larval zebrafish brain
}

https://doi.org/10.1523/ENEURO.0337-21.2022

Cite as: eNeuro 2022; 10.1523/ENEURO.0337-21.2022

Received: 16 August 2021

Revised: 13 January 2022

Accepted: 21 January 2022

This Early Release article has been peer-reviewed and accepted, but has not been through the composition and copyediting processes. The final version may differ slightly in style or formatting and will contain links to any extended data.

Alerts: Sign up at www.eneuro.org/alerts to receive customized email alerts when the fully formatted version of this article is published.

Copyright @ 2022 Pinion et al.

This is an open-access article distributed under the terms of the Creative Commons Attribution 4.0 International license, which permits unrestricted use, distribution and reproduction in any medium provided that the original work is properly attributed. 


\title{
1. Differential electrographic signatures generated by mechanistically-diverse seizurogenic
} compounds in the larval zebrafish brain

\section{LFP's of seizurogenic compounds in the zebrafish brain}

3. Authors: Joseph Pinion ${ }^{1}$, Callum Walsh², Marc Goodfellow ${ }^{3}$, Andrew D. Randall ${ }^{2}$, Charles R. Tyler ${ }^{1}$, Matthew J. Winter ${ }^{1 *}$

\author{
Affiliations \\ ${ }^{1}$ Biosciences, College of Life and Environmental Sciences, University of Exeter, Exeter, Devon, EX44QD, \\ United Kingdom. \\ ${ }^{2}$ Medical School, University of Exeter, Exeter, Devon, EX4 4PS, United Kingdom. \\ ${ }^{3}$ Living Systems Institute and Mathematics, College of Engineering, Mathematics and Physical \\ Sciences, University of Exeter, Exeter, Devon, EX4 4QF, United Kingdom.
}

\section{Author contributions}

JP: Conceptualization, Methodology, Software, Writing - Original Draft, Formal analysis, Writing Review \& Editing CW: Methodology, Software, Formal analysis, Writing - Review \& Editing MG: Methodology, Software, Formal analysis, Writing - Review \& Editing ADR: Conceptualization, Formal analysis, Writing - Review \& Editing, Supervision, Funding acquisition CRT: Conceptualization, Writing

- Review \& Editing, Supervision, Funding acquisition MJW: Conceptualization, Writing - Review \& Editing, Supervision, Funding acquisition.

\author{
5. Joseph Pinion \\ Biosciences, College of Life and Environmental Sciences, \\ University of Exeter, M05, Geoffrey Pope Building, \\ Stocker Rd, Exeter, Devon, EX4 4QD, United Kingdom.
}


Tel: 01392726258

Email: jp422@exeter.ac.uk
6. 7
7. 2
8. 0
9. 217
10. 11.803
12. 1929

\section{Acknowledgements}

The authors would like to thank the staff in the Aquatic Resources Centre at the University of Exeter, for the maintenance of the zebrafish broodstock, and Dr Misha Ahrens for originally supplying the elav/3:GCaMP6s fish line. This work was funded by the National Centre for the Replacement, Refinement and Reduction of Animals in Research (NC3Rs) through a Ph.D. Studentship awarded to JP (NC/R001421/1), and the University of Exeter, College of Life and Environmental Sciences. The authors would also like to thank Dr Jonathan Brown for his critical evaluation of the final draft manuscript.

14. No

15. National Centre for the Replacement, Refinement and Reduction of Animals in Research (NC3Rs) through a Ph.D. Studentship awarded to JP (NC/R001421/1). 


\section{Abstract}

We assessed similarities and differences in the electrographic signatures of local field potentials evoked by different pharmacological agents in zebrafish larvae. We then compared and contrasted these characteristics with what is known from electrophysiological studies of seizures and epilepsy in mammals, including humans. Ultimately, our aim was to phenotype neurophysiological features of drug-induced seizures in larval zebrafish for expanding knowledge on the translational potential of this valuable alternative to mammalian models. Local field potentials were recorded from the midbrain of 4-day old zebrafish larvae exposed to a pharmacologically diverse panel of seizurogenic compounds, and the outputs of these recordings were assessed using frequency domain analysis. This included analysis of changes occurring within various spectral frequency bands of relevance to mammalian CNS circuit pathophysiology. From these analyses, there were clear differences in the frequency spectra of drug-exposed local field potentials, relative to controls, many of which shared notable similarities with the signatures exhibited by mammalian CNS circuits. These similarities included the presence of specific frequency components comparable to those observed in mammalian studies of seizures and epilepsy. Collectively, the data presented provide important information to support the value of larval zebrafish as an alternative model for the study of seizures and epilepsy. These data also provide further insight into the electrophysiological characteristics of seizures generated in non-mammalian species by the action of neuroactive drugs.

\section{Significance Statement}

In this study we offer novel insight into the frequency domain of the local field potentials (LFPs) for a range of seizurogenic compounds in zebrafish larvae. We make a direct comparison of seizurogenic compounds with varying mechanisms of action and, where possible, compare the effects of these compounds in zebrafish larvae with those recorded in mammals in terms of the frequency components of their LFPs. This study adds to the mounting body of evidence supporting the use of the larval zebrafish as a powerful alternative model organism for seizure and epilepsy research. 


\section{Introduction}

Seizures are defined as periods of excessive or hyper-synchronous brain activity (Fisher et al.,

3 2014a) which, when recurrent and unprovoked, define the chronic disease epilepsy (Falco-Walter et

4 al., 2018). In addition, seizure occurrences and myoclonus appear to be a feature of the

5 pathophysiology of a number of other CNS diseases, including Alzheimer's disease and other forms of

6 dementia (Beagle et al., 2017). Seizures themselves have a wide-ranging aetiology that includes a

7 substantial proportion attributable to the adverse action of drugs. It has been estimated, for example,

8 that approximately $6 \%$ of new-onset seizures and $9 \%$ of status epilepticus cases are as a result of drug toxicity (Chen et al., 2016), which includes the inadvertent action of multiple marketed drugs (Easter et al., 2009; Ruffmann et al., 2006). Seizures themselves can be definitively identified in human patients and non-clinical animal models using neurophysiological assessment techniques such as local field potential (LFP) recordings and electroencephalography (EEG) (Lévesque and Avoli, 2019; Usman et al., 2019). Using these techniques, seizures can be observed to present diverse electrographic dynamics, with some common components including low voltage fast activity, e.g. high frequency oscillations (HFOs) (Jiménez-Jiménez et al., 2015; Wang et al., 2020) in the fast ripple frequency bands (250-500 Hz), or high amplitude periodic spikes (Jiménez-Jiménez et al., 2015; Perucca et al., 2014; Wang et al., 2020). However, precisely how, and where, they are manifest can vary between different forms of epilepsy and different causes of seizure (Jiménez-Jiménez et al., 2015; Perucca et al., 2014; Wang et al., 2020).

In order to better understand why seizures occur and how to treat epilepsy the use of experimental models is important. Clearly such models should include an appropriate, often complex neural architecture, and the best models are those performed in vivo, preferably in the absence of confounds generated by anaesthesia. With this in mind, a drive towards more ethical and costeffective approaches for studying complex neurological disorders has increased interest in alternative, non-mammalian, vertebrate models for such studies. Of these models, the larval zebrafish offers considerable potential as a highly genetically tractable alternative for screening epilepsy related genes 
and for the study of genetic mutation-induced spontaneous seizures (Baraban et al., 2013a; Griffin et al., 2021). To date, zebrafish have been relatively widely used for assessing drug-induced neural activity, and various studies have described the effects of seizurogenic chemicals on their behaviour (Afrikanova et al., 2013; Alfaro et al., 2011; Baraban et al., 2013b; Berghmans et al., 2007; Winter et al., 2021), electrophysiology (Afrikanova et al., 2013; Baraban et al., 2013b, 2007; Cho et al., 2017; Copmans et al., 2019; Griffin et al., 2017; Hortopan et al., 2010; Hunyadi et al., 2017; Kim et al., 2010b; Liu and Baraban, 2019), and functional imaging phenotypes (Burgstaller et al., 2019; Burrows et al., 2020; Ghannad-Rezaie et al., 2019; Winter et al., 2021, 2017). Electrophysiological assessments, in particular, offer extremely high temporal resolution and allow direct comparison of zebrafish-derived data with that obtained from neurophysiological assessments undertaken in mammals. Consequently, the recording of larval zebrafish electrophysiological data offers the opportunity to directly compare abnormal electrographic dynamics between these two taxonomically diverse sets of model organisms. In larval zebrafish, typically, LFP recordings from small clusters of neurons in easily identified anatomical targets (such as the optic tectum) are used to measure the response of the brain to drug treatment. Indeed, LFP recordings have been used to assess the electrographic response of the larval zebrafish to a few seizurogenic compounds including picrotoxin, pilocarpine (Baraban, 2013; Hortopan et al., 2010), and, most often, pentylenetetrazole (PTZ) (Baraban et al., 2007; Copmans et al., 2019; Kim et al., 2010b; Liu and Baraban, 2019). Despite the relatively widespread use of electrophysiology in larval zebrafish, little is actually known about the specific electrographic characteristics of seizures induced by diverse chemicals in this model organism, other than the aforementioned exceptions. There are few published studies which characterise LFP profiles in larval zebrafish across a range of excitatory mechanisms, for example, or that have compared their characteristics with those used to define seizures in more traditional models of seizures and epilepsy using mammalian electrophysiology and human EEG data. Here we sought to address this knowledge gap by assessing the LFP-based response of 4 days post-fertilisation (dpf) larval zebrafish, to treatment with a variety of seizurogenic compounds which act through a range of pharmacological mechanisms 
of action. Using frequency domain analysis on the resulting data we aimed to assess the similarities and differences in the electrographic signatures of LFP's between different pharmacologies, and to compare and contrast these characteristics with what is known from electrophysiological studies of seizures and epilepsy in mammals including humans, thereby adding considerably to our knowledge of the translational value of this model.

\section{Methods}

The experimental approach used is detailed in the following sections and the process is summarised in Fig. 1A. Throughout, the data shown in graphs and tables are the means, \pm SEM of data points from individual animals (n). All analyses were performed using MATLAB (MATLAB version 9.8.0, 2020), including the signal processing toolbox 8.4 (MATLAB signal processing toolbox 8.4, 2020) and wavelet toolbox 5.4 (MATLAB wavelet toolbox 5.4, 2020).

\subsection{Experimental animals}

For this work, 4dpf transgenic zebrafish, of unknown sex, with a pan-neuronal $\mathrm{Ca}^{2+}$ sensor (elav/3:GCaMP6s) were used. This transgenic line allowed us to compare the electrographic recording data generated here, with functional imaging data obtained from a separate study (Winter et al., 2021). 4dpf larval zebrafish were selected as these are not considered protected vertebrates under European animal legislation and as such are ethically preferable to the use of older, protected, animals. The use of $4 \mathrm{dpf}$ larval zebrafish also enabled the use of neuromuscular blocking agents and for electrophysiological recordings to be performed in the absence of a potentially confounding general anesthetic.

Adult elavl3:GCaMP6s broodstock (Ahrens et al., 2014) (kindly supplied by Dr Misha Ahrens, Janelia Research Campus, Howard Hughes Medical Institute, Ashburn, Virginia, USA) were held in

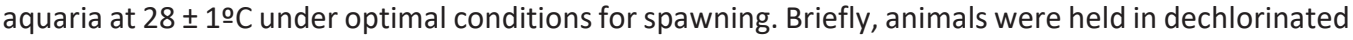
mains tap water (referred to here as 'culture water'), which was routinely monitored for water quality 
parameters. Fertilized eggs were collected shortly after spawning and transferred to Petri dishes which were filled with culture water and held at $28 \pm 1$ 으 until use in experiments at $4 \mathrm{dpf}$ (see (Winter et al., 2017) for full details). All work was undertaken under project and personal licences granted by the UK Home Office under the UK Animals (Scientific Procedures) Act.

\subsection{Test compounds and concentration range selection}

Test compounds were selected based upon their known seizurogenic potential in mammals, including humans, as defined by case studies of seizure incidence and assays in model organisms as outlined in Table 1. Appropriate exposure concentration ranges ensuring CNS responsiveness without generalised toxicity were also selected based upon previously published data (Winter et al., 2021). The exposure conditions adopted are summarised in Table 1. All compounds were dissolved in extracellular solution (ECS) and $\mathrm{pH}$ adjusted to $~ 7.5$ prior to use. The composition of ECS was: $1 \mathrm{mM}$ $\mathrm{NaCl}, 2.9 \mathrm{mM} \mathrm{KCl}, 10 \mathrm{mM}$ HEPES, $1.2 \mathrm{mM} \mathrm{MgCl}$, $10 \mathrm{mM}$ Dextrose, and $2.1 \mathrm{mM} \mathrm{CaCl}_{2}$ (Baraban, 2013). For each compound, there were 3 exposure concentrations, and for each experimental group, 7-8 larvae were used per treatment group. All test chemicals and other reagents were obtained from Sigma-Aldrich (Gillingham, UK) or Tocris (Bristol, UK).

\subsection{In vivo local field potential recordings from zebrafish brains}

Individual 4dpf larvae were paralysed using $4 \mathrm{mM}$ tubocurarine and then positioned dorsal side up in the recording chamber in $1.5 \%$ low melting point agarose containing ECS. Throughout recording the mounted fish were kept in a static bath containing ECS. Under $4 \mathrm{x}$ magnification, a glass $2 \mathrm{M} \mathrm{NaCl}$ filled microelectrode (resistance of 3-5 $\mathrm{M} \Omega$ ) was inserted into the midbrain to record extracellular LFPs from a small network of neurons (Figure 1B). The area chosen for electrode placement was based on clear anatomical landmarks, and the electrode was placed a small distance (circa 30 $\mu \mathrm{m}$ ) lateral from the midline of the brain. This was decided upon to ensure consistency of electrode placement in a specific brain area. Recordings were captured using an Axon CNS Multiclamp 700B amplifier and 
digitised using an Axon Digidata 1440A (Amplifier settings were Mode: I=0, Gain: 20, Bessel: 2 kHz), and data were recorded with the Clampex 10.4 software. Following introduction of the recording electrode, larvae were equilibrated for 300 seconds prior to beginning data acquisition. The LFP recording procedure consisted of acquiring 7.5 minutes of baseline data following which the test compound was added to the ECS by pipette, followed by recording in the presence of compound for 17.5 minutes. The exposure epoch was defined as the period between 17.5 and 25 minutes, thus allowing 10 minutes for the test compound to penetrate the larva and reach equilibrium. All raw electrophysiological signals were recorded at $10 \mathrm{kHz}$, downsampled to $2 \mathrm{kHz}$ and digitally filtered, using a Butterworth filter, with high pass at $1 \mathrm{~Hz}$ and low pass at $500 \mathrm{~Hz}$, to filter out spiking activity from individual neurons while preserving LFP. Throughout recording and pre-equilibration larva were flashed with two flashes of blue $(488 \mathrm{~nm})$ light, each lasting $100 \mathrm{~ms}$ and separated by a $500 \mathrm{~ms}$ gap, repeated every 4.5 seconds. This was undertaken for two purposes: to provide visual stimulation that could be detected using electrophysiology so as to ensure healthy nervous system functioning and to provide regular periodic CNS stimulation by a standard sensory pathway. Moreover, previous studies have successfully used light stimulation protocols to encourage spontaneous seizures in zebrafish expressing genes related to Dravet syndrome, thus we hypothesised it may also help to additionally sensitise animals to drug treatment (Eimon et al., 2018). Following completion of the compound exposure recording period, larval heart rate was visually assessed to confirm fish health. Additional vehicle-only experiments (identical except for no test compound exposure) were performed at regular intervals during the data acquisition phase to ensure that any changes in LFPs were not associated with the time/date of assessment.

\subsection{Data analysis: comparisons of area under the curve of the LFP baseline and exposure period}

As a first step in our analyses we aimed to assess if the test compounds had an effect on the overall brain activity levels, over time. Seizures are associated with excessive hypersynchronous activity, therefore fish exposed to seizurogenic drugs might be expected to display a change in overall 
signal amplitude. The area under the curve (AUC) was calculated, in 30 second time bins, for the absolute value of the Hilbert transform of the filtered LFP signal to allow an assessment of the 'volume' of activity over time. This allowed us to visualise the time-course of oscillatory activity, whether it was increasing or decreasing throughout the exposure or baseline periods see Figure 5A. Moreover, the total AUC over the baseline and exposure period was also calculated and a Wilcoxon signed rank test (corrected for multiple comparisons using Benjamini and Hochberg method (Benjamini and Hochberg, 1995)) was used to identify differences between baseline and drug-exposure recording epochs, see Figure 5B.

\subsection{Data analysis: spectral analysis}

Among the most commonly used approaches for assessing changes in neural activity patterns is frequency domain analysis, which allows investigation into the contribution of oscillations at different frequencies to the overall signal. Commonly in human EEG or mammalian animal model LFP datasets, oscillations are binned into bands of behavioural relevance, and changes in the power of these bands can correlate with transitions in behavioural or cognitive state (Başar et al., 2001). While the same frequency bands may not be as behaviourally-relevant in zebrafish, we hypothesised that due to the similar underlying neurophysiology of mammals and zebrafish, interpreting spectral changes in the context of the same bands may provide useful insights into underlying mechanisms of seizurogenesis. Furthermore, performing such analysis in the $4 \mathrm{dpf}$ larval zebrafish served to provide a shared context which, it was hypothesised, would be important for making interspecies comparisons of electrophysiological responses to seizurogenic chemical treatment. Fourier transforms were performed on the signal from baseline and exposure periods of each fish, and the resulting spectra during each exposure period were normalised to the corresponding spectra of the baseline recordings. Next, the mean normalised spectra for each fish were averaged within treatment groups (see Figure 6B). Finally, normalised spectra were binned into frequency bands used for studying mammalian and human EEG data (Moffett et al., 2017; Wang et al., 2020). These bands were: $\delta(1-4 \mathrm{~Hz}), \theta(4-7 \mathrm{~Hz})$, 
$\alpha / \mu(8-13 \mathrm{~Hz}), \beta$ (beta, 15-30 Hz), $\nu$ (gamma, 30-80 Hz) and high $\gamma(80-150 \mathrm{~Hz})$ and HFO $(150-500$ $\mathrm{Hz}$ ). The activity in each band was then compared between test compound-treated and control fish using Mann-Whitney U-tests corrected for multiple comparisons using the Benjamini and Hochberg method (Benjamini and Hochberg, 1995) see Figure 6A and Table 2.

\subsection{Data analysis: classical multidimensional scaling (MDS) of baseline normalised spectral data}

In order to visualise the similarity or differences between representative spectra for each treatment group, classical multi-dimensional scaling was performed across the average normalised power spectra for each fish. Subsequently, the mean of the first two coordinates was calculated across treatments groups, and were plotted. The eigenvalues for these coordinates accounted for $>74 \%$ of the sum of all the eigenvalues.

\subsection{Data analysis: Event detection}

The analysis described above was derived from averaging the activity over long periods. However we also wanted to examine the presence of differences in the LFP on shorter time scales, akin to examining the morphology of epileptiform spikes or rhythm. To achieve this, we algorithmically selected shorter time periods based on differences from baseline in their frequency components, which we defined as 'events'. These events were defined as periods of time 1 second long whose frequency spectra deviated significantly from the baseline. Each larva has a 'drug free' baseline period and 'events' that were considered significantly different from baseline were selected. In the case of control larva this 'exposure period' was also a drug free baseline period. However, due to the sensitivity of the equipment used there were still some 'events' that differed enough from the baseline in the control fish to be included, although compared to the drug treated fish, these were very few in number (see figure 8). These 'erroneous' events are likely to be the result of electrical noise common in these kinds of recordings. For event detection, initially wavelet transformations were performed on the recorded voltage timeseries for both the exposure and the baseline periods in order to produce 
highly temporally resolved frequency spectra. For this a continuous 1-D wavelet transform was used, which was obtained using the analytic Morse wavelet with the symmetry parameter (gamma) equal to 3 , and the time-bandwidth product equal to 60 , using 10 voices per octave. The frequencies of the resultant wavelets were binned into the following bands: $1-4 \mathrm{~Hz}, 4-7 \mathrm{~Hz}, 8-13 \mathrm{~Hz}, 15-30 \mathrm{~Hz}, 30-80$ $\mathrm{Hz}, 80-150 \mathrm{~Hz}$, and $150-500 \mathrm{~Hz}$ (see Figure 2A). These bands broadly correlate with neural frequency bands used in mammalian models (Moffett et al., 2017; Wang et al., 2020). Next, the average over time in each of these frequency bands was calculated across the entire baseline period. Subsequently the Euclidean distance between the frequency spectra of every time-point in the wavelets and the average baseline frequency spectra was calculated. This allowed identification of where in the wavelet transformations deviations from the mean of the baseline occurred. After plotting the resultant Euclidean distances as shown in Figure 2B, we used MATLAB's 'findpeaks' function to identify where the Euclidean distances were greater than two standard deviations from the mean of the baseline period, selecting 1 second long events. Next, taking all of the events selected using this first pass approach, the event wavelets for each fish were plotted separately in Euclidean space. From this, only the exposure events that were further from the centroid of the baseline events than the most distant baseline event, were selected. This process ensured we selected only the events whose spectra were distinct from those that occurred during the drug-free baseline period. The numbers of these events were calculated for each larva and compared between control and treatment groups using a Wilcoxon rank sum test, corrected for multiple comparisons using the Benjamini and Hochberg method (Benjamini and Hochberg, 1995).

\subsection{Data analysis: Wavelet of selected events}

In order to better understand how the temporal profile of events differed between compounds, event epochs were selected from each treatment group of each compound. The events with the smallest Euclidean distance from the mean spectra of all the events selected for that treatment group (using the method outlined above), were selected as best representing events produced by that 
treatment. In addition to the presentation of the filtered timeseries, a heatmap of the wavelet transforms was also generated, as shown in Figure 3.

\section{Code Accessibility}

The code/software described in the paper is freely available online at: https://github.com/jp908/Ephys-Seizure-ZF. The code is available as Extended Data.

\section{Results}

\subsection{Changes in AUC between baseline and exposure}

The results of the changes in AUC from baseline to exposure are summarised in Figure 5. Only picrotoxin ( $p=0.0081$, corrected- $p=0.0104, W=51, z$-value $=-2.6461)$ and SB205607 ( $p=0.0006$, corrected-p=0.0015, $\mathrm{W}=270, \mathrm{z}$-value $=3.4286$ ) exposure resulted in significant changes in AUC between the baseline and exposure periods, exhibiting increased and decreased AUC respectively. The AUC of picrotoxin appeared to oscillate slightly across 30 second time bins with a slight upward gradient.

\subsection{Spectral Frequency Band Analysis}

The results of the spectral analysis of the in vivo LFP data are summarised in Figure 6 and table

2. From these data, it can be seen that exposure to the highest concentrations of chlorpromazine and picrotoxin resulted in a significant increase in activity in the slower delta-frequency band compared with the control animals. In the higher frequency $\gamma$, high $\gamma$, and HFO bands, significant increases in activity were observed after exposure to RST, picrotoxin and aminophylline. Interestingly, exposure to chlorpromazine resulted in significantly reduced, $\beta, \gamma$, high $\gamma$, and HFO band activity at all of the concentrations tested, and this was not observed for any other compounds assessed here. Exposure to SB205607 and donepezil resulted in no significant changes in any of the frequency bands measured. In addition, exposure to picrotoxin resulted in increases in $\alpha$ band oscillatory activity, while aminophylline and picrotoxin exposure resulted in increases in $\beta$ power. Notably, picrotoxin resulted in an increase in power in every single frequency band, but only at the highest concentration. 


\subsection{Classical multi-dimensional scaling}

Classical multi-dimensional scaling of the spectral data revealed the distribution of each treatment group in 2-dimensions. The outer regions of the resultant scatter plot (Figure 7) are occupied by the highest concentration of chlorpromazine, donepezil, RST and picrotoxin. Notably there was overlap of the middle concentration of aminophylline and picrotoxin, likely due to similar increases in $\beta, \gamma$ and high frequency oscillations. SB205607 and the lowest concentration of aminophylline lie closest to the control reflecting the relative absence of induced effects on the parameters measured. The highest concentration of chlorpromazine is located furthest from RST and picrotoxin likely reflecting its reduction in oscillations at $>15 \mathrm{~Hz}$. Furthermore, RST and picrotoxin are located on the right hand side of the scatterplot likely due to their notable induced increases in the magnitude of oscillations at $>15 \mathrm{~Hz}$.

\subsection{Number of Detected Events}

Exposure to aminophylline, chlorpromazine, donepezil and RST appeared to increase the number of events detected in a dose-dependent manner (see figure 8). The highest concentrations of, donepezil $(p=0.0354 \quad z$-value $=-2.764$, test statistic $=239.5)$, picrotoxin $(p=0.0273, \quad z$-value $=-2.715$, test statistic=234), and RST ( $p=0.0159, z$-value $=--3.220$, test statistic=229.5) resulted in a significant increase in the number of detected events as compared to the control.

\subsection{Mean Event and Wavelet Analysis}

The results of the mean event and wavelet analysis are summarised in Figure 3. Exposure to the compounds tested resulted in bursts of rhythmic activity of specific frequencies consistent with changes in the spectra observed in the previous analyses (Figure 6). Chlorpromazine's wavelet spectrograms, for example, showed a distinct amelioration of $>80 \mathrm{~Hz}$ activity particularly in the higher concentrations, in addition to bursts of theta, delta and alpha range activity. The events identified in 
animals exposed to donepezil were categorised by a gradual polarisation followed by a large low frequency event in the $<15 \mathrm{~Hz}$ range with an additional high frequency component at the peak of, and subsequent to, the main depolarisation. However, there was also evidence of bursting type high frequency activity. Picrotoxin exposure resulted in wavelet spectrograms characterised by a high amplitude activity in the $80 \mathrm{~Hz}$ or less frequency range, and appeared to show a distinct dosedependent increase in activity. RST exposed animals were observed to contain a bursting type of high frequency activity in addition to sharp spike-type discharges, as illustrated most clearly at the middle concentration. Notably RST appeared to lack much of the lower frequency component. Both aminophylline and SB205607 appeared to induce medium sized events, although notably the highest concentration of aminophylline appeared to have some similarities with donepezil's high concentration event profile.

\section{Discussion}

Using in vivo mid-brain LFP recordings in 4dpf larval zebrafish, we demonstrate responsiveness to a range of pharmacological agents implicated in the induction of seizures in mammals. The data we present show that exposure to chlorpromazine (a phenothiazine antipsychotic), donepezil (an acetylcholinesterase inhibitor), picrotoxin (a GABA $A_{A}$ antagonist) and RST (an NMDAR agonist) results in quantifiable, concentration-dependent, altered neuronal electrophysiology. Furthermore, the electrographic responses exhibited differed between each drug, and in some cases exhibited characteristics commensurate with the induction of interictal and ictal electrophysiology phenotypes similar to those classified in humans (Fisher et al., 2014b).

\subsection{Electrographic Phenotypes of Representative Traces}



aim of our study was to explore what 'abnormal' LFP recordings might emerge following exposure of zebrafish larvae to drugs acting via known seizurogenic mechanisms. Furthermore, from these phenotypes, we aimed to classify such patterns in the context of their frequency components, and to compare these components to human EEG data and models of seizure in mammals. To this end we compared the representative traces that were selected using our event detection algorithm with specific examples of pre-ictal, inter-ictal and ictal type waveforms, as reported by Fisher et al (Fisher et al., 2014b). The representative trace for the highest concentration of chlorpromazine, for example, appeared to comprise of rhythmical evolving theta, delta and alpha frequencies, which is an electrophysiological phenotype commonly seen preceding seizure in EEG traces recorded clinically (Fisher et al., 2014b). Similarly, the high amplitude, high frequency, discharges observed in the representative traces for the intermediate exposure concentrations for both donepezil and RST share similarities with intracerebral stereo-EEG recordings of seizures reported in mammals (Fisher et al., 2014b). These discharges also contained very high frequency $(80-500 \mathrm{~Hz})$ components similar to examples of seizures reported previously in mammals (Gliske et al., 2017; Jiruska et al., 2017, 2013; Mackenzie et al., 2002; Shiri et al., 2015; Wang et al., 2020). The representative traces for the highest concentration of aminophylline, in addition to that seen for the lowest and highest concentrations of donepezil, appear consistent with electrodecromental EEG patterns, a electrophysiological phenotype commonly seen preceding seizure in EEG traces recorded clinically (Fisher et al., 2014b). aminophylline, donepezil and picrotoxin in our study, with traces of spontaneous ictal and inter-ictal activity identified by (Griffin et al., 2021) in their recording of LFPs in zebrafish (where they were screening for potentially seizurogenic genetic modifications), showed strong similarities. Moreover, the traces for the medium and high concentrations of RST appeared similar to their definition of an ictal-type waveform. These similarities support a good degree of consistency between drug induced seizures and spontaneous seizures, although a more extensive comparison would be necessary to 
311 confirm this. Compared with behavioural and imaging-based assessments of seizures in zebrafish

$312(13,22,26,34-39)$, in vivo LFP recordings provide a direct measure of the electrographic response of

313 model organisms to neuroactive drug treatment. This is important as the data generated more readily

314 allows comparisons between model species (see Table 3), a feature which is crucial for evaluating the

315 translational power of the zebrafish as a surrogate for understanding the effects of neuroactive drugs

316 in higher vertebrates, including humans.

\section{$317 \quad 4.2$ Trace Amplitude and Seizure}

Given that seizures are considered to be high amplitude events, measuring the change in the average AUC induced by the different compounds was initially undertaken. Notably the largest increase in AUC was observed after exposure to picrotoxin which is commonly used in animal models as a seizure precipitant (Baraban, 2013; Fan et al., 2019; Ridler et al., 2018; Winter et al., 2008). This is consistent with previous studies in zebrafish exposed to picrotoxin that reported the presence of high amplitude events (Baraban, 2013). Interestingly, however, the NMDA-receptor agonist RST, which is also a well-known model convulsant, induced no change in AUC despite inducing changes in other measured parameters. It is worth noting that seizure like states are often characterised by periods of bursting activity with intermittent quiescence (Barnett et al., 2013). In this context it is possible that periods of seizure like activity induced via RST are bursting in nature, with long intervals in-between and thus not best detected using our protocol. In support of this, upon a more detailed analysis of the spectral components of its electrographic signature, the resultant data suggested that RST exposure was associated with elevated neural activity in specific frequency-bands, rather than showing a more generalised elevated activity (see below). In a previous study using $\mathrm{Ca}^{2+}$ imaging (Winter et al., 2021), it was also reported that there was a relative absence of oscillatory activity after exposure to RST (and the pharmacologically similar NMDA). Given that here we saw elevated activity only in the $>15 \mathrm{~Hz}$ frequency range (see below), and notwithstanding the sensitivity of $\mathrm{Ca}^{2+}$ dye based imaging, it is possible that in the previous study, the temporal resolution of the imaging-based approach used was insufficient to capture such rapid oscillations thus highlighting the relative 
advantages and disadvantages of each type of approach when studying drug-induced neuronal events.

\subsection{Neural Oscillations Zebrafish versus Mammals}

The binning of generated frequency spectra into specific frequency bands is a commonly used approach in the analysis of mammalian electrophysiological data (Başar et al., 2001). We adopted this approach primarily to directly compare neural activity signatures between $4 \mathrm{dpf}$ larval zebrafish and mammalian models of drug-induced seizures. It should be noted that we consider the changes seen in the different frequency bands for all drugs to be as a result of bursts of rhythmic activity. This means there are both periodic and aperiodic contributions to the frequency spectra. We believe this is the case, because the event detection algorithm utilised here identified bursts of rhythmic activity whose spectra were consistent with the frequency spectra performed on the full recordings. Moreover, the number of these events was not adequate to constitute the full traces. As such our analyses suggested that drug exposure resulted in transient bursts of rhythmic activity that altered the overall spectral profile of the recordings, as opposed to sustained oscillatory activity.

In multiple EEG studies undertaken in mice, the antipsychotic medications haloperidol and clozapine have been shown to significantly reduce gamma power (Olszewski et al., 2013; Sun et al., 2020). Here, we also observed a significant reduction in all of the frequency bands of $>15 \mathrm{~Hz}$ upon exposure to chlorpromazine. Both chlorpromazine and haloperidol broadly reduce monoamine signalling, a neurotransmitter sub-group that is highly conserved between larval zebrafish and mammals in both their molecular underpinnings and behavioural functionality (Maximino et al., 2016). Indeed, haloperidol has been shown to induce locomotor impairments in zebrafish, an effect mirrored in the common side effects in humans (e.g. drowsiness, dizziness and neuromuscular dysfunction (Giacomini et al., 2006)). Chlorpromazine has also been shown to induce significant alterations in 4dpf larval brain functional connectivity (Winter et al., 2021) and our data here also show comparable oscillatory activity induced by this antipsychotic compound between zebrafish and mammals. The confirmation here of shared brain oscillatory changes, in addition to the aforementioned similar behavioural manifestations, suggests that the established links between neural frequency bands and 
mammalian behaviour may have some translatability to zebrafish.

Exposure to the acetylcholinesterase inhibitor donepezil increased power in the theta frequency bands in larval zebrafish. In mammals theta and gamma oscillations are associated with successful memory recall (Barnett et al., 2013). Notably, a mechanistically similar compound and cholinesterase inhibitor, physostigmine, has been shown to reverse scopolamine-induced learning impairment in zebrafish, suggesting effectiveness in reversing impaired cognition (Kim et al., 2010a). In this context it would be interesting to assess the behavioural response of zebrafish to donepezil specifically given the apparent enhancement of theta oscillations in the electrophysiology data we present here. Indeed, observation of donepezil-induced cognitive enhancement in larval zebrafish would provide further evidence of a similar relationship between brain oscillatory patterns and behavioural manifestations in larval zebrafish as those observed in mammals.

Perturbations in oscillations in the gamma and high gamma ranges are common across a variety of psychiatric disorders, including attention deficit hyperactivity disorder, schizophrenia and, of particular relevance here, epilepsy (Herrmann and Demiralp, 2005). Epilepsy specifically is associated with an increase in gamma oscillations likely due to cortical excitation (Herrmann and Demiralp, 2005). Indeed, in human EEGs, oscillations in the gamma and fast gamma range precede interictal epileptiform spikes in the seizure onset zone (Jiruska et al., 2013). Similarly, in the nucleus accumbens of rats, there are increases in gamma power when seizure kindling is induced using kainic acid (Ma and Leung, 2010). Here we observed significant increases in gamma and fast gamma activity after exposure to the two seizure precipitants picrotoxin and RST, in addition to aminophylline. Previously published work in mice has shown that treatment with picrotoxin has been shown to increase gamma oscillations in the olfactory bulb, an effect that could be suppressed via inhibition of ionotropic glutamate receptors (Lepousez and Lledo, 2013). In addition, in rats, NMDA agonists induce acetylcholine release and affect an increase in both beta and gamma oscillations in the basal forebrain (Fournier et al., 2004). In our study, both picrotoxin and RST appeared to induce abnormal electrographic events, similar to those recorded in previous studies in zebrafish (Afrikanova et al., 
2013; Baraban et al., 2005; Copmans et al., 2019; Hortopan et al., 2010; Löscher, 2017) and consistent with seizures observed in human EEG (Fisher et al., 2014b). Notably our data suggest that these events, much like in humans and rats, were characterised by an increase in gamma and high gamma frequency oscillations. The fact that chlorpromazine appeared to uniquely reduce gamma and high gamma oscillations is interesting and raises the issue of primary versus secondary pharmacological activity and dosing levels. Certainly, chlorpromazine is known to have potent sedative effects linked to its well-known $\mathrm{H}_{1}$ activity (von Coburg et al., 2009), and is typically associated with a seizurethreshold lowering effect rather than a direct seizurogenic effect as measured using our exposure method (Chi et al., 2017).

The highest frequency band we measured, in the range between 150 and $500 \mathrm{~Hz}$, contained high frequency oscillations (HFOs) (Wang et al., 2020). HFOs are strongly correlated with the epileptogenic zone and have been implicated as a useful biomarker of epilepsy (Lévesque and Avoli, 2019), although their use to prospectively define the epileptogenic zone is not clear (Jacobs et al., 2018). Indeed, fast ripples $(250-500 \mathrm{~Hz})$ appear to be highly indicative of epileptic tissue in both human conditions and animal models (Jiruska et al., 2017). HFOs are believed to occur as a result of the synchronisation of fast firing within populations of interconnected neurons generating high-frequency population spikes which, when recorded extracellularly, present as an HFO event (Jiruska et al., 2017). Individual Pyramidal neurons cannot fire fast enough to account for oscillations higher than $300 \mathrm{~Hz}$, thus, fast ripple oscillations have been proposed to be generated via the action of synchronised, but out-of-phase, neurons (Jiruska et al., 2013). Here, a significant increase in the magnitude of HFOs was observed after exposure to aminophylline, picrotoxin and RST, while chlorpromazine appeared to reduce the magnitude of HFOs. This is consistent with studies in mouse brain slices showing induction of HFOs via picrotoxin exposure (Shiri et al., 2015). Moreover, automated event detection designed to categorize events unique from baseline identified several events across these compounds that contained HFOs, including after exposure to donepezil, RST and picrotoxin across all concentrations.

\subsection{Study Limitations}


Exposure to the $\delta$-opioid agonist, SB205607, showed little evidence of altered brain electrophysiology despite showing significantly elevated activity in a number of brain regions at 500

$417 \mu \mathrm{M}$ (the top concentration used here) in an imaging based assessment undertaken in 4dpf zebrafish 418 (Winter et al., 2021). Other studies have reported insensitivity of the larval zebrafish to specific $\delta O R$ agonists such as SNC80 (Rodriguez and Gonzalez-Nunez, 2006) although this does not explain why previous work with SB205607 suggested some degree of neuroactivity (Winter et al., 2021). One possible reason for this could be explained by the relative strengths and weaknesses of electrophysiological versus imaging-based neural functional assessments. Electrophysiological assessment offers unparalleled levels of temporal resolution, whereas imaging based approach offer much greater spatial coverage. Given the LFP approach used here measures electrical activity in a small population of neurons in one part of the brain it is possible that this region was not activated by SB205607 exposure. Certainly this would be supported by the data from our $\mathrm{Ca}^{2+}$ imaging work (Winter et al., 2021) which suggested comparatively low levels of activity in mid brain regions compared with the hind brain. This could be investigated further by the use of multi-electrode arrays to allow for greater spatial coverage during LFP recordings (e.g. (Hong et al., 2016)). mammalian electrophysiology data, there are limitations to the larval zebrafish as a model. Specifically, in vitro LFP recordings taken in mammals are often performed on specific anatomical structures which in the case of seizure and epilepsy research are recognised as hyper-excitatory in nature, such as the hippocampus. In zebrafish larvae, recording from specific brain structures is extremely challenging due the small size of the brain, thus recordings are generally taken from the midbrain or the forebrain with little ability to differentiate between specific sub regions of higher or lower relevance. In this respect, the use of genetically modified models in which specific neural circuits or populations are labelled may prove extremely useful and allow more precise placement of electrodes in structures that have been identified as especially appropriate for measuring the type of brain activity being investigated. 
In summary, in vivo LFP-mediated assessment of neural activity in $4 \mathrm{dpf}$ zebrafish larvae revealed

responsiveness to seizurogenic compounds that act via a range of pharmacological mechanisms of

action. Furthermore, the resultant electrographic profiles exhibited by $4 \mathrm{dpf}$ zebrafish larvae exposed

to a number of these compounds show clear differences in their characteristics and in some cases

share notable similarities with the signatures exhibited by mammalian, including human,

electrophysiological profiles. The data generated here add to the body of data supporting the use of

the larval zebrafish as a complimentary and potentially alternative model for the study of seizures and generated in non-mammalian species.

\section{Declaration of competing interests}

All authors declare no potentially competing interests.

\section{$\underline{\text { References }}$}

458 Afrikanova T, Serruys A-SK, Buenafe OEM, Clinckers R, Smolders I, de Witte PAM, Crawford AD, Esguerra C V. (2013) Validation of the Zebrafish Pentylenetetrazol Seizure Model: Locomotor versus Electrographic Responses to Antiepileptic Drugs. PLoS One 8:e54166.

Ahmad F, Noldus LPJJ, Tegelenbosch RAJ, Richardson MK (2012) Zebrafish embryos and larvae in behavioural assays. Behaviour 149:1241-1281.

Ahnaou A, Huysmans H, Jacobs T, Drinkenburg WHIM (2014) Cortical EEG oscillations and network connectivity as efficacy indices for assessing drugs with cognition enhancing potential. Neuropharmacology 86:362-377. 
(2014) Light-sheet functional imaging in fictively behaving zebrafish. Nat Methods 11:883-884.

Ahrens MB, Orger MB, Robson DN, Li JM, Keller PJ (2013) Whole-brain functional imaging at cellular resolution using light-sheet microscopy. Nat Methods 10:413-420.

Albertson TE, Stark LG, Joy RM, Bowyer JF (1983) Aminophylline and kindled seizures. Exp Neurol 81:703-713.

Alfaro JM, Ripoll-Gómez J, Burgos JS (2011) Kainate administered to adult zebrafish causes seizures similar to those in rodent models. Eur J Neurosci 33:1252-1255.

Baraban SC (2013) Forebrain Electrophysiological Recording in Larval Zebrafish. J Vis Exp 71:2-5.

Baraban SC, Dinday MT, Castro PA, Chege S, Guyenet S, Taylor MR (2007) A Large-scale Mutagenesis Screen to Identify Seizure-resistant Zebrafish. Epilepsia 48:1151-1157.

Baraban SC, Dinday MT, Hortopan GA (2013a) Drug screening in Scn1a zebrafish mutant identifies clemizole as a potential Dravet syndrome treatment. Nat Commun 4:1-10.

Baraban SC, Dinday MT, Hortopan GA (2013b) Drug screening in Scn1a zebrafish mutant identifies clemizole as a potential Dravet syndrome treatment. Nat Commun 4.

Baraban SC, Taylor MR, Castro PA (2005) PENTYLENETETRAZOLE INDUCED CHANGES IN ZEBRAFISH BEHAVIOR , NEURAL ACTIVITY AND C-FOS EXPRESSION. Neuroscience 131:759-768.

Barnett W, O’Brien G, Cymbalyuk G (2013) Bistability of silence and seizure-like bursting. J Neurosci Methods 220:179-189.

Başar E, Başar-Eroglu C, Karakaş S, Schürmann M (2001) Gamma, alpha, delta, and theta oscillations govern cognitive processes. Int J Psychophysiol 39:241-248.

Beagle A, Darwish S, Ranasinghe K, Karageorgiou A, Vossel K (2017) Relative Incidence of Seizures and Myoclonus in Alzheimer's Disease, Dementia with Lewy Bodies, and Frontotemporal Dementia. Physiol Behav 176:139-148.

Benjamini Y, Hochberg Y (1995) Controlling the False Discovery Rate : A Practical and Powerful Approach to Multiple Testing. J R Stat Soc 57:289-300.

Berghmans S, Hunt J, Roach A, Goldsmith P (2007) Zebrafish offer the potential for a primary screen 
to identify a wide variety of potential anticonvulsants. Epilepsy Res 75:18-28.

Bloechliger M, Rüegg S, Jick SS, Meier CR, Bodmer M (2015) Antipsychotic Drug Use and the Risk of Seizures: Follow-up Study with a Nested Case-Control Analysis. CNS Drugs 29:591-603.

Burgstaller J, Hindinger E, Donovan J, Maschio MD, Kist AM, Gesierich B, Portugues R, Baier H (2019) Light-sheet imaging and graph analysis of antidepressant action in the larval zebrafish brain network. bioRxiv 618843 .

Burrows DRW, Samarut, Liu J, Baraban SC, Richardson MP, Meyer MP, Rosch RE (2020) Imaging epilepsy in larval zebrafish. Eur J Paediatr Neurol 24:70-80.

Chen HY, Albertson TE, Olson KR (2016) Treatment of drug-induced seizures. Br J Clin Pharmacol 81:412-419.

Chi SH, Jeong HG, Lee S, Oh SY, Kim SH (2017) Effects of psychotropic drugs on seizure threshold during electroconvulsive therapy. Psychiatry Investig 14:647-655.

Cho S-J, Byun D, Nam T-S, Choi S-Y, Lee B-G, Kim M-K, Kim S (2017) Zebrafish as an animal model in epilepsy studies with multichannel EEG recordings. Sci Rep 7:3099.

Chu N -S (1981) Caffeine- and Aminophylline-Induced Seizures. Epilepsia 22:85-94.

Copmans D, Kildgaard S, Rasmussen SA, Ślezak M, Dirkx N, Partoens M, Esguerra C V., Crawford AD, Larsen TO, De Witte PAM (2019) Zebrafish-based discovery of antiseizure compounds from the north sea: Isoquinoline alkaloids TMC-120A and TMC-120B. Mar Drugs 17:1-20.

Druschky K, Bleich S, Grohmann R, Engel RR, Neyazi A, Stübner S, Toto S (2019) Seizure rates under treatment with antipsychotic drugs: Data from the AMSP project. World J Biol Psychiatry 20:732-741.

Easter A, Bell ME, Damewood JR, Redfern WS, Valentin JP, Winter MJ, Fonck C, Bialecki RA (2009) Approaches to seizure risk assessment in preclinical drug discovery. Drug Discov Today.

Eimon PM, Ghannad-Rezaie M, De Rienzo G, Allalou A, Wu Y, Gao M, Roy A, Skolnick J, Yanik MF (2018) Brain activity patterns in high-throughput electrophysiology screen predict both drug efficacies and side effects. Nat Commun 9. 
Falco-Walter JJ, Scheffer IE, Fisher RS (2018) The new definition and classification of seizures and epilepsy. Epilepsy Res 139:73-79.

Fan J, Thalody G, Kwagh J, Burnett E, Shi H, Lewen G, Chen SJ, Levesque P (2019) Assessing seizure liability using multi-electrode arrays (MEA). Toxicol Vitr 55:93-100.

Fisher RS et al. (2014a) ILAE Official Report: A practical clinical definition of epilepsy. Epilepsia 55:475-482.

Fisher RS, Scharfman HE, deCurtis M (2014b) How Can We Identify Ictal and Interictal Abnormal Activity? 3-23.

Fournier GN, Materi LM, Semba K, Rasmusson DD (2004) Cortical acetylcholine release and electroencephalogram activation evoked by ionotropic glutamate receptor agonists in the rat basal forebrain. Neuroscience 123:785-792.

Ghannad-Rezaie M, Eimon PM, Wu Y, Yanik MF (2019) Engineering brain activity patterns by neuromodulator polytherapy for treatment of disorders. Nat Commun 10.

Giacomini NJ, Rose B, Kobayashi K, Guo S (2006) Antipsychotics produce locomotor impairment in larval zebrafish. Neurotoxicol Teratol 28:245-250.

Gliske S V., Stacey WC, Lim E, Holman KA, Fink CG (2017) Emergence of Narrowband High Frequency Oscillations from Asynchronous, Uncoupled Neural Firing. Int J Neural Syst 27:1-11.

Griffin A, Carpenter C, Liu J, Paterno R, Grone B, Hamling K, Moog M, Dinday MT, Figueroa F, Anvar M, Ononuju C, Qu T, Baraban SC (2021) Phenotypic analysis of catastrophic childhood epilepsy genes. Commun Biol 4.

Griffin A, Hamling KR, Knupp K, Hong SG, Lee LP, Baraban SC (2017) Clemizole and modulators of serotonin signalling suppress seizures in Dravet syndrome. Brain 140:669-683.

Herrmann CS, Demiralp T (2005) Human EEG gamma oscillations in neuropsychiatric disorders. Clin Neurophysiol 116:2719-2733.

Hillman EM, Voleti V, Patel K, Li W, Yu H, Perez-Campos C, Benezra SE, Bruno RM, Galwaduge PT (2018) High-speed 3D imaging of cellular activity in the brain using axially-extended beams and 
light sheets. Curr Opin Neurobiol 50:190-200.

Hong SG, Lee P, Baraban SC, Lee LP (2016) A Novel Long-term, Multi-Channel and Non-invasive Electrophysiology Platform for Zebrafish. Sci Rep 6:1-10.

Hortopan GA, Dinday MT, Baraban SC (2010) Zebrafish as a model for studying genetic aspects of epilepsy. DMM Dis Model Mech 3:144-148.

Hunyadi B, Siekierska A, Sourbron J, Copmans D, de Witte PAM (2017) Automated analysis of brain activity for seizure detection in zebrafish models of epilepsy. J Neurosci Methods 287:13-24.

Jacobs J, Wu JY, Perucca P, Zelmann R, Mader M, Dubeau F, Mathern GW, Schulze-Bonhage A, Gotman J (2018) Removing high-frequency oscillations: A prospective multicenter study on seizure outcome. Neurology 91:e1040-e1052.

Jiménez-Jiménez D, Nekkare R, Flores L, Chatzidimou K, Bodi I, Honavar M, Mullatti N, Elwes RDC, Selway RP, Valentín A, Alarcón G (2015) Prognostic value of intracranial seizure onset patterns for surgical outcome of the treatment of epilepsy. Clin Neurophysiol 126:257-267.

Jiruska P, Alvarado-Rojas C, Schevon CA, Staba R, Stacey W, Wendling F, Avoli M (2017) Update on the mechanisms and roles of high-frequency oscillations in seizures and epileptic disorders. Epilepsia 58:1330-1339.

Jiruska P, de Curtis M, Jefferys JGR, Schevon CA, Schiff SJ, Schindler K (2013) Synchronization and desynchronization in epilepsy: Controversies and hypotheses. J Physiol 591:787-797.

Kim YH, Lee Y, Kim D, Jung MW, Lee CJ (2010a) Scopolamine-induced learning impairment reversed by physostigmine in zebrafish. Neurosci Res 67:156-161.

Kim YH, Lee Y, Lee K, Lee T, Kim YJ, Lee CJ (2010b) Reduced neuronal proliferation by proconvulsant drugs in the developing zebrafish brain. Neurotoxicol Teratol 32:551-557.

Kumlien E, Lundberg PO (2010) Seizure risk associated with neuroactive drugs: Data from the WHO adverse drug reactions database. Seizure 19:69-73.

Lepousez G, Lledo P-M (2013) Odor Discrimination Requires Proper Olfactory Fast Oscillations in Awake Mice. Neuron 80:1010-1024. 
Lévesque M, Avoli M (2019) High-frequency oscillations and focal seizures in epileptic rodents. Neurobiol Dis 124:396-407.

Liu J, Baraban SC (2019) Network properties revealed during multi-scale calcium imaging of seizure activity in Zebrafish. eNeuro 6.

Löscher W (2017) Animal Models of Seizures and Epilepsy: Past, Present, and Future Role for the Discovery of Antiseizure Drugs. Neurochem Res 42:1873-1888.

Ma J, Leung LS (2010) Kindled seizure in the prefrontal cortex activated behavioral hyperactivity and increase in accumbens gamma oscillations through the hippocampus. Behav Brain Res 206:6877.

Mackenzie L, Medvedev A, Hiscock JJ, Pope KJ, Willoughby JO (2002) Picrotoxin-induced generalised convulsive seizure in rat: Changes in regional distribution and frequency of the power of electroencephalogram rhythms. Clin Neurophysiol 113:586-596.

MATLAB signal processing toolbox 8.4 (2020) R2020a The Mathworks Inc., Natick, Massachusetts.

MATLAB version 9.8 .0 (2020) R2020a The Mathworks Inc., Natick, Massachusetts.

MATLAB wavelet toolbox 5.4 (2020) R2020a The Mathworks Inc., Natick, Massachusetts.

Maximino C, P. Costa B, G. Lima M (2016) A Review of Monoaminergic Neuropsychopharmacology in Zebrafish, 6 Years Later: Towards Paradoxes and their Solution. Curr Psychopharmacol 5:96138.

Moffett SX, O'Malley SM, Man S, Hong D, Martin J V. (2017) Dynamics of high frequency brain activity. Sci Rep 7:4-8.

Olszewski M, Piasecka J, Goda SA, Kasicki S, Hunt MJ (2013) Antipsychotic compounds differentially modulate high-frequency oscillations in the rat nucleus accumbens: A comparison of first- and second-generation drugs. Int J Neuropsychopharmacol 16:1009-1020.

Perucca P, Dubeau F, Gotman J (2014) Intracranial electroencephalographic seizure-onset patterns: Effect of underlying pathology. Brain 137:183-196.

Ridler T, Matthews P, Phillips KG, Randall AD, Brown JT (2018) Initiation and slow propagation of 
epileptiform activity from ventral to dorsal medial entorhinal cortex is constrained by an inhibitory gradient. J Physiol 596:2251-2266.

Rodriguez RE, Gonzalez-Nunez V (2006) Characterization of Opioid-binding sites in zebrafish brain. J Pharmacol Exp Ther 316:900-904.

Ruffmann C, Bogliun G, Beghi E (2006) Epileptogenic drugs: A systematic review. Expert Rev Neurother 6:575-589.

Schoepp DD, Smith CL, Lodge D, Millar JD, Leander JD, Sacaan AI, Lunn WHW (1991) D,L-(Tetrazol-5yl) glycine: a novel and highly potent NMDA receptor agonist. Eur J Pharmacol 203:237-243.

Schwartz M, Scott D. (1974) Aminophylline-Induce Seizures. Epilepsia 15:501-505.

Shiri Z, Manseau F, Lévesque M, Williams S, Avoli M (2015) Interneuron activity leads to initiation of low-voltage fast-onset seizures. Ann Neurol 77:541-546.

Sun D, Kermani M, Hudson M, He X, Unnithan RR, French C (2020) Effects of antipsychotic drugs and potassium channel modulators on cognition-related local field potential spectral properties in mouse hippocampus and frontal cortex. bioRxiv.

Usman SM, Khalid S, Akhtar R, Bortolotto Z, Bashir Z, Qiu H (2019) Using scalp EEG and intracranial EEG signals for predicting epileptic seizures: Review of available methodologies. Seizure 71:258-269.

von Coburg Y, Kottke T, Weizel L, Ligneau X, Stark H (2009) Potential utility of histamine H3 receptor antagonist pharmacophore in antipsychotics. Bioorganic Med Chem Lett 19:538-542.

Wang Y, Zhou D, Yang X, Xu X, Ren L, Yu T, Zhou W, Shao X, Yang Z, Wang S, Cao D, Liu C, Kwan SY, Xiang J (2020) Expert consensus on clinical applications of high-frequency oscillations in epilepsy. Acta Epileptol 2.

Wilcoxon rank sum test - MATLAB ranksum - MathWorks United Kingdom [WWW Document] (n.d.). URL https://uk.mathworks.com/help/stats/ranksum.html (accessed 7.29.21).

Winter M.J., Pinion J., Tochwin A., Takesono A., Ball J.S., Grabowski P., Metz J., Trznadel M., Tse K., Redfern W.S., Hetheridge M.J., Goodfellow M., Randall A.D. TCR (2021) Neuropharmacological 
profiling using functional brain imaging in larval zebrafish. Br J Pharmacol.

Winter MJ, Pinion J, Tochwin A, Takesono A, Ball JS, Grabowski P, Metz J, Trznadel M, Tse K, Redfern WS, Hetheridge MJ, Goodfellow M, Randall AD, Tyler CR (2021) Functional brain imaging in larval zebrafish for characterising the effects of seizurogenic compounds acting via a range of pharmacological mechanisms. Br J Pharmacol 9:1-19.

Winter MJ, Redfern WS, Hayfield AJ, Owen SF, Valentin J, Hutchinson TH (2008) Validation of a larval zebrafish locomotor assay for assessing the seizure liability of early-stage development drugs. J Pharmacol Toxicol Methods 57:176-187.

Winter MJ, Windell D, Metz J, Matthews P, Pinion J, Brown JT, Hetheridge MJ, Ball JS, Owen SF, Redfern WS, Moger J, Randall AD, Tyler CR (2017) 4-Dimensional Functional Profiling in the Convulsant-Treated Larval Zebrafish Brain. Sci Rep 7:1-16.

Yajima Y, Narita M, Takahashi-Nakano Y, Misawa M, Nagase H, Mizoguchi H, Tseng LF, Suzuki T (2000) Effects of differential modulation of $\mu$-, $\delta$ - and $k$-opioid systems on bicuculline-induced convulsions in the mouse. Brain Res 862:120-126. 
Table 1) Test compounds and exposure concentration ranges used for in vivo electrophysiological recording in 4dpf elavl3:GCaMP6s zebrafish larvae. Also shown are published evidence to support seizurogenicity. All compounds were sourced from Sigma-Aldrich (Gillingham, UK) or Tocris (Bristol, 664 UK).

Figure 1) A schematic showing the experimental process and example data from in vivo electrophysiological recording in 4dpf elavl3:GCaMP6s zebrafish larvae. Panel A) The experimental process used. Panel B) Left: A paralysed, mounted zebrafish larva with glass electrode inserted into midbrain. The Red circle indicates the placement of the tip of the electrode, while the blue dashed line indicates landmarks used to consistently place the electrode. Right: Representative LFP recording from midbrain of zebrafish larva at baseline and after administration of $30 \mathrm{mM}$ picrotoxin.

Figure 2) Example data obtained from in vivo electrophysiological recording in a $4 \mathrm{dpf}$ elavl3:GCaMP6s zebrafish larva exposed to donepezil A) A representative frequency binned wavelet transformation from a single larva, the top graph shows the baseline and the bottom wavelet transform after drug administration. These transformations were used to identify events from the full timeseries. B) A plot of the Euclidean distance of each time point from the average of the baseline. The black dotted line represents the threshold for selecting events. All peaks above this line were selected and used to find events which were subsequently clustered.

Figure 3) Example of an event plus 2 seconds either side for each treatment group. For each treatment group, the timeseries' are displayed for each event with a wavelet transform below each one displaying the frequency domain over the same time period. The events selected were the events whose spectra were closest in Euclidean distance to the mean event spectra for that treatment group, 
shows the colour scaling for the magnitude of the wavelet transformation. Each column represents a different concentration set and each row represents a different compound.

Figure 4) Mean number of events detected per treatment group. Bar graph showing the mean number of events per treatment group. Error bars represent the SEM ( $n=7-8)$. *adjacent to the bars indicate a statistically significant difference from control $(p<0.05)$ using a Wilcoxon rank sum test corrected for multiple comparisons using the Benjamini and Hochberg method.

Figure 5) Analysis of the area under the curve (AUC) of the Hilbert transform of the LFP recordings. Panel A) Shows the mean baseline normalised AUC of the absolute value of the Hilbert transform of the LFP averaged into 30 second time bins. The shadows represent the SEM ( $n=20-24)$. The left hand side of the graph represents the baseline period, while the right-hand side represents the exposure period. The gap in time from 7-17 minutes is time allotted for compounds to take effect. Panel B) Shows the baseline normalised AUC for each of the compounds tested. The bars show the Normalised AUC averaged across all treatment groups for each compound, while the normalised AUC of individual larvae are represented as transparent grey circles. Data are shown as the mean with error bars depicting the SEM $(n=20-24)$ * indicates a statistically significant difference between baseline and exposure periods $(p<0.05)$ using Wilcoxon signed rank test and corrected for multiple comparisons using the Benjamini and Hochberg method (Benjamini and Hochberg, 1995).

Figure 6) Data generated for larvae exposed to each of the test compounds after spectral analysis and categorisation into specific frequency bands. Panel A) The bars show the baseline normalised mean amplitude for each of the neural frequency bands frequently used for categorising mammalian electrophysiological data (Moffett et al., 2017; Wang et al., 2020). Data are shown as the mean with error bars showing the SEM (n=7-20). The baseline normalised power for individual larvae are represented by the transparent grey circles. *indicates a statistically significant difference from control $(p<0.05)$ using Mann Whitney U-tests and corrected for multiple comparisons using the Benjamini and Hochberg method (Benjamini and Hochberg, 1995). Panel B) shows the mean baseline normalised power spectra for each compound treatment group. In this case the $\mathrm{x}$-axis is scaled to the common logarithm. Shading represents the SEM for each data point $(n=7-20)$ across the power spectra. Black lines here indicate the mean of the control animal power spectra, and therefore are the same for each graph.

Table 2) All statistical test outputs from frequency band analyses: The activity in each frequency band compared between test compound-treated and control fish using unpaired Mann-Whitney U-test and corrected for multiple comparisons using the Benjamini and Hochberg method (Benjamini and Hochberg, 1995). The columns from left to right contain the relevant frequency band, compound, concentration of exposure, p-value, Benjamini and Hochberg corrected p-value, Rank Sum test statistic and corresponding z-statistic computed when the method is 'approximate'. All statistics were undertaken using the MatLab statistics and machine learning toolbox ("Wilcoxon rank sum test MATLAB ranksum - MathWorks United Kingdom," n.d.).

Figure 7) Multidimensional Scaling of the normalised power spectra: Right-hand image: A scatter graph of the first two coordinates produced after classical multi-dimensional scaling of the mean normalised power spectra of each treatment group (see Figure 6B): Left-hand image: A scatter graph of the first two coordinates produced after classical multidimensional scaling of each individual larva. In both plots the circles represent the lowest concentration, the triangles the middle concentration and the diamonds the highest concentration used for each treatment group. The black square represents the control group. 
Table 3) Summary of information comparing the effect of selected drugs on EEG in mammals from

738 published data with the data obtained for zebrafish in this study.

739 
Table 1

741

\begin{tabular}{|c|c|c|c|}
\hline Compound Name & Pharmacodynamics & Concentrations Used & Seizure liability \\
\hline Aminophylline & $\begin{array}{l}\text { Adenosine Receptor } \\
\text { Antagonist and } \\
\text { phosphodiesterase } \\
\text { inhibitor }\end{array}$ & $1 \mathrm{mM}, 2 \mathrm{mM}, 4 \mathrm{mM}$ & $\begin{array}{l}\text { Various cases of seizure in } \\
\text { humans, and evidence of } \\
\text { kindling in rats (Albertson } \\
\text { et al., 1983; Schwartz and } \\
\text { Scott, 1974). }\end{array}$ \\
\hline Chlorpromazine & $\begin{array}{l}\text { Dopamine, } \\
\text { Serotonin, } \\
\text { Histamine, } \\
\text { Muscarinic and } \alpha 1 \text { - } \\
\text { and } \alpha 2 \text {-adrenergic } \\
\text { receptor antagonist }\end{array}$ & $\begin{array}{l}31.25 \mu \mathrm{M}, 62.5 \mu \mathrm{M} \\
125 \mu \mathrm{M}\end{array}$ & $\begin{array}{l}\text { Increased risk of seizure in } \\
\text { patients receiving anti- } \\
\text { psychotic drug (APD) } \\
\text { treatment, particularly } \\
\text { polytherapy. Seizure risk 5- } \\
\text { fold higher in individuals } \\
\text { receiving low/medium } \\
\text { potency APDS such as } \\
\text { chlorpromazine } \\
\text { (Bloechliger et al., 2015; } \\
\text { Druschky et al., 2019). }\end{array}$ \\
\hline Donepezil & $\begin{array}{l}\text { Acetylcholinesterase } \\
\text { inhibitor }\end{array}$ & $\begin{array}{l}125 \mu \mathrm{M}, 62 \mu \mathrm{M} \text {, } \\
31 \mu \mathrm{M}\end{array}$ & $\begin{array}{l}\text { Among the top } 10 \text { drugs } \\
\text { most commonly } \\
\text { associated with seizures in } \\
\text { world health organisation } \\
\text { adverse drug reaction } \\
\text { database (Kumlien and } \\
\text { Lundberg, 2010). }\end{array}$ \\
\hline Picrotoxin & $\begin{array}{l}\mathrm{GABA}_{A} \text { receptor } \\
\text { Antagonist }\end{array}$ & $\begin{array}{l}30 \mu \mathrm{M}, 60 \mu \mathrm{M}, \\
120 \mu \mathrm{M}\end{array}$ & $\begin{array}{l}\text { Commonly used } \\
\text { convulsant compound } \\
\text { used for modelling } \\
\text { seizures (Mackenzie et al., } \\
\text { 2002). }\end{array}$ \\
\hline $\begin{array}{l}\text { (RS)-(Tetrazol-5- } \\
\text { yl)glycine }\end{array}$ & $\begin{array}{l}\text { NMDA receptor } \\
\text { Agonist }\end{array}$ & $\begin{array}{l}62.5 \mu \mathrm{M}, 125 \mu \mathrm{M}, \\
250 \mu \mathrm{M}\end{array}$ & $\begin{array}{l}\text { Convulsant compound } \\
\text { used for modelling } \\
\text { seizures (Schoepp et al., } \\
\text { 1991). }\end{array}$ \\
\hline SB205607 (TAN-67) & $\begin{array}{l}\delta \text {-opioid receptor } \\
\text { agonist }\end{array}$ & $\begin{array}{l}125 \mu \mathrm{M}, 250 \mu \mathrm{M}, 500 \\
\mu \mathrm{M}\end{array}$ & $\begin{array}{l}\text { Increases incidence of } \\
\text { convulsions in bicuculline } \\
\text { kindled rats (Yajima et al., } \\
\text { 2000). }\end{array}$ \\
\hline
\end{tabular}


Table 2

\begin{tabular}{|c|c|c|c|c|c|c|}
\hline $\begin{array}{c}\text { Frequency } \\
\text { Band }\end{array}$ & Compound & Concentration & P-Value & $\begin{array}{l}\text { Corrected } \\
\text { P-Value }\end{array}$ & $\begin{array}{l}\text { Test } \\
\text { Statistic }\end{array}$ & Z-Value \\
\hline$\delta$ & Chlorpromazine & $62.5 \mu \mathrm{M}$ & 0.013812 & 0.03942 & 235 & -2.46211 \\
\hline$\delta$ & Chlorpromazine & $125 \mu \mathrm{M}$ & 0.010224 & 0.035258 & 239 & -2.56817 \\
\hline$\delta$ & Picrotoxin & $120 \mu \mathrm{M}$ & 0.013812 & 0.038106 & 235 & -2.46211 \\
\hline$\theta$ & Picrotoxin & $120 \mu \mathrm{M}$ & 0.002566 & 0.013276 & 225 & -3.01539 \\
\hline$\alpha$ & Picrotoxin & $120 \mu \mathrm{M}$ & 0.000544 & 0.003464 & 217 & -3.45802 \\
\hline$\beta$ & Aminophylline & $2 \mathrm{mM}$ & 0.010089 & 0.036306 & 233 & -2.57277 \\
\hline$\beta$ & Chlorpromazine & $31.25 \mu \mathrm{M}$ & 0.011825 & 0.037643 & 340 & 2.517311 \\
\hline$\beta$ & Chlorpromazine & $62.5 \mu \mathrm{M}$ & 0.00438 & 0.018126 & 332 & 2.849409 \\
\hline$\beta$ & Chlorpromazine & $125 \mu \mathrm{M}$ & 0.011825 & 0.036249 & 340 & 2.517311 \\
\hline$\beta$ & Picrotoxin & $120 \mu \mathrm{M}$ & 0.000442 & 0.003329 & 216 & -3.51335 \\
\hline$\gamma$ & Aminophylline & $2 \mathrm{mM}$ & 0.002566 & 0.012495 & 225 & -3.01539 \\
\hline$\gamma$ & Chlorpromazine & $31.25 \mu \mathrm{M}$ & 0.0001 & 0.002071 & 367 & 3.890389 \\
\hline v & Chlorpromazine & $62.5 \mu \mathrm{M}$ & 0.000188 & 0.002223 & 348 & 3.734663 \\
\hline$\gamma$ & Chlorpromazine & $125 \mu \mathrm{M}$ & 0.000053 & 0.004369 & 370 & 4.042954 \\
\hline$\gamma$ & Picrotoxin & $120 \mu \mathrm{M}$ & 0.00029 & 0.002668 & 214 & -3.62401 \\
\hline$\gamma$ & RST & $250 \mu \mathrm{M}$ & 0.011825 & 0.034954 & 240 & -2.51731 \\
\hline High $\gamma$ & Aminophylline & $2 \mathrm{mM}$ & 0.002566 & 0.011801 & 225 & -3.01539 \\
\hline High $\gamma$ & Aminophylline & $4 \mathrm{mM}$ & 0.011821 & 0.039135 & 234 & -2.51744 \\
\hline High $\gamma$ & Chlorpromazine & $31.25 \mu \mathrm{M}$ & 0.000081 & 0.002236 & 368 & 3.941244 \\
\hline High $\gamma$ & Chlorpromazine & $62.5 \mu \mathrm{M}$ & 0.000188 & 0.001945 & 348 & 3.734663 \\
\hline High $\gamma$ & Chlorpromazine & $125 \mu \mathrm{M}$ & 0.000053 & 0.002184 & 370 & 4.042954 \\
\hline High $\gamma$ & Picrotoxin & $120 \mu \mathrm{M}$ & 0.000442 & 0.003052 & 216 & -3.51335 \\
\hline High $\gamma$ & RST & $250 \mu \mathrm{M}$ & 0.00293 & 0.012763 & 231 & -2.975 \\
\hline HFO & Aminophylline & $2 \mathrm{mM}$ & 0.001466 & 0.008088 & 222 & -3.18138 \\
\hline HFO & Aminophylline & $4 \mathrm{mM}$ & 0.008586 & 0.032303 & 232 & -2.6281 \\
\hline HFO & Chlorpromazine & $31.25 \mu \mathrm{M}$ & 0.0001 & 0.001657 & 367 & 3.890389 \\
\hline HFO & Chlorpromazine & $62.5 \mu \mathrm{M}$ & 0.000995 & 0.00588 & 340 & 3.292036 \\
\hline HFO & Chlorpromazine & $125 \mu \mathrm{M}$ & 0.000123 & 0.0017 & 366 & 3.839535 \\
\hline $\mathrm{HFO}$ & Picrotoxin & $120 \mu \mathrm{M}$ & 0.00029 & 0.002401 & 214 & -3.62401 \\
\hline HFO & RST & $250 \mu \mathrm{M}$ & 0.004766 & 0.018784 & 234 & -2.82244 \\
\hline
\end{tabular}




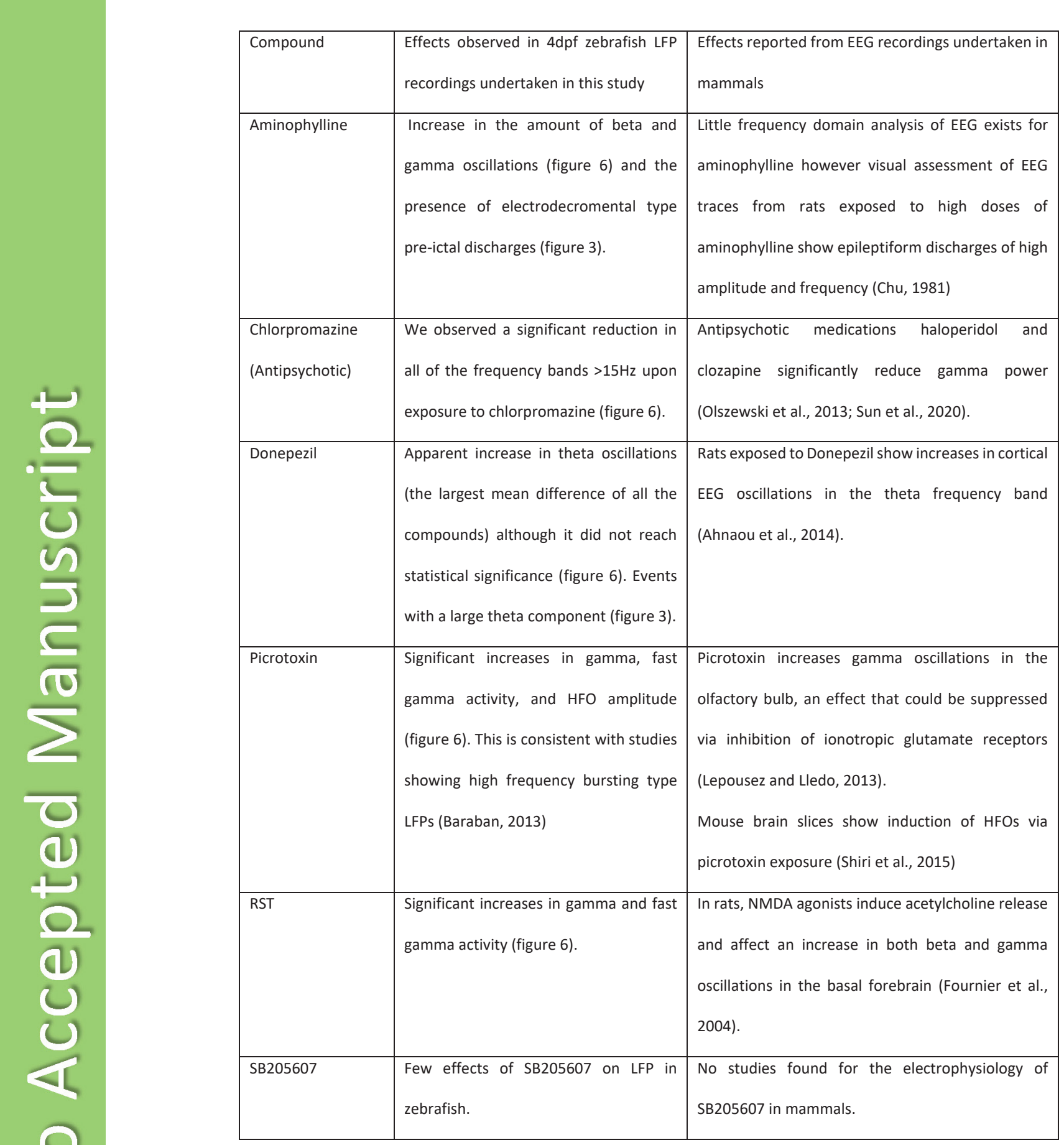


Mount fish in LMP agarose

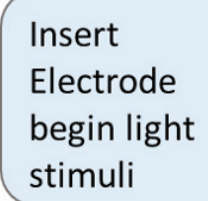

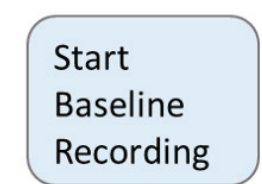

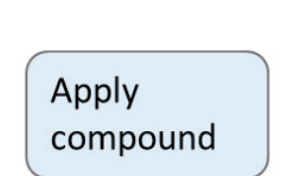

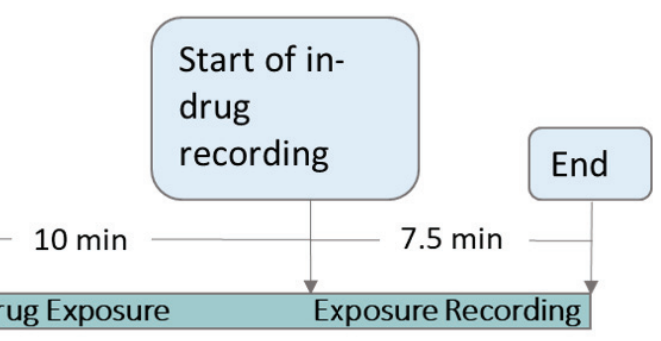
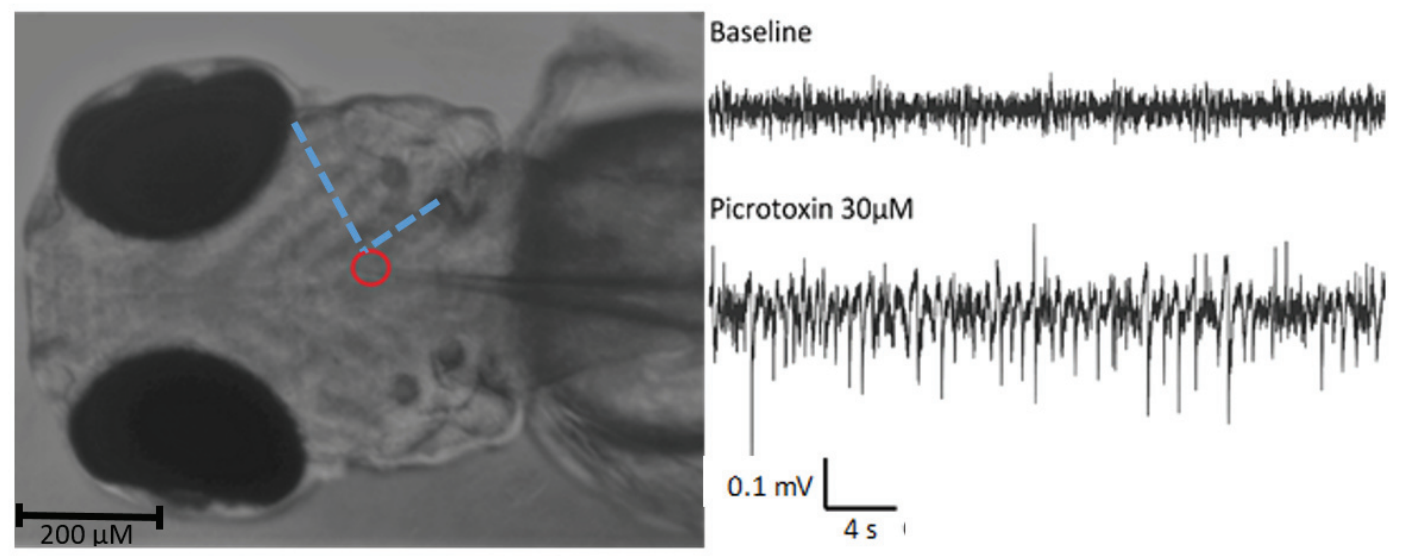


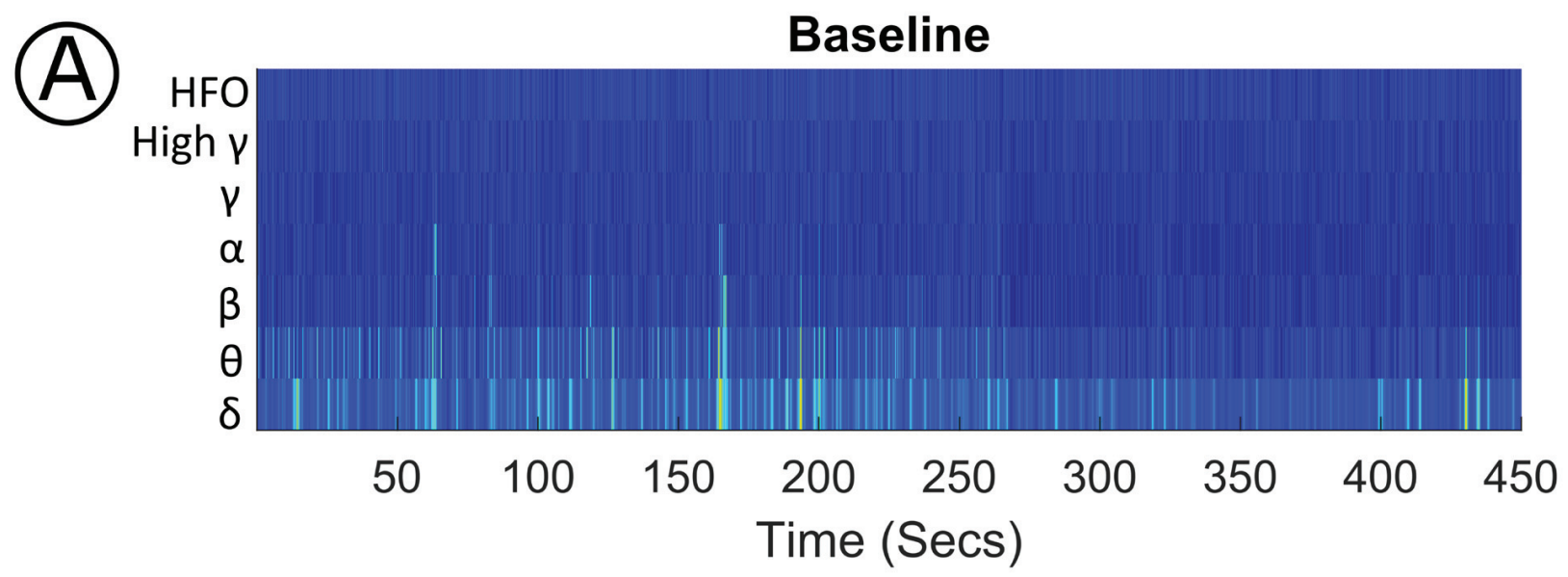

Exposure

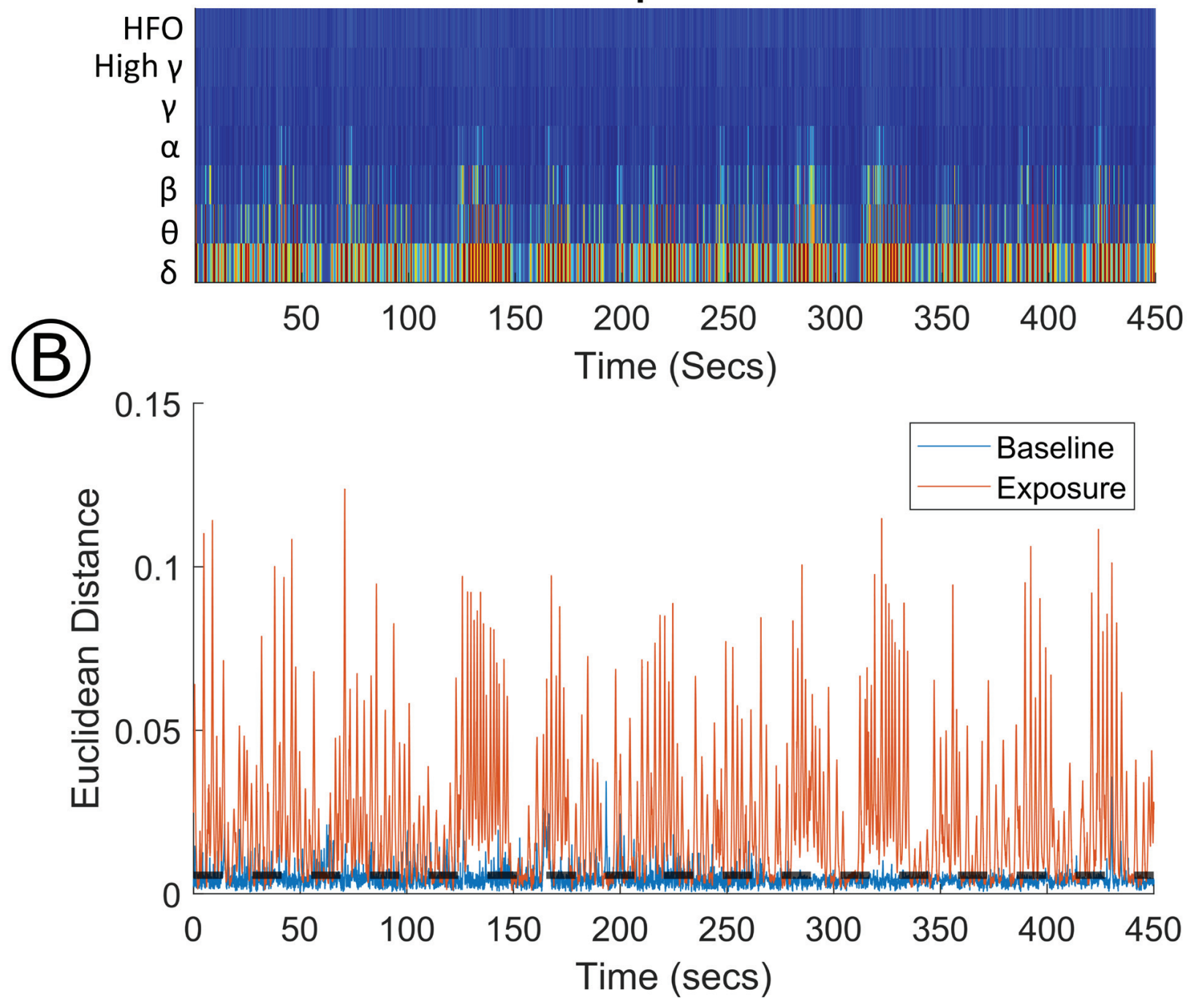




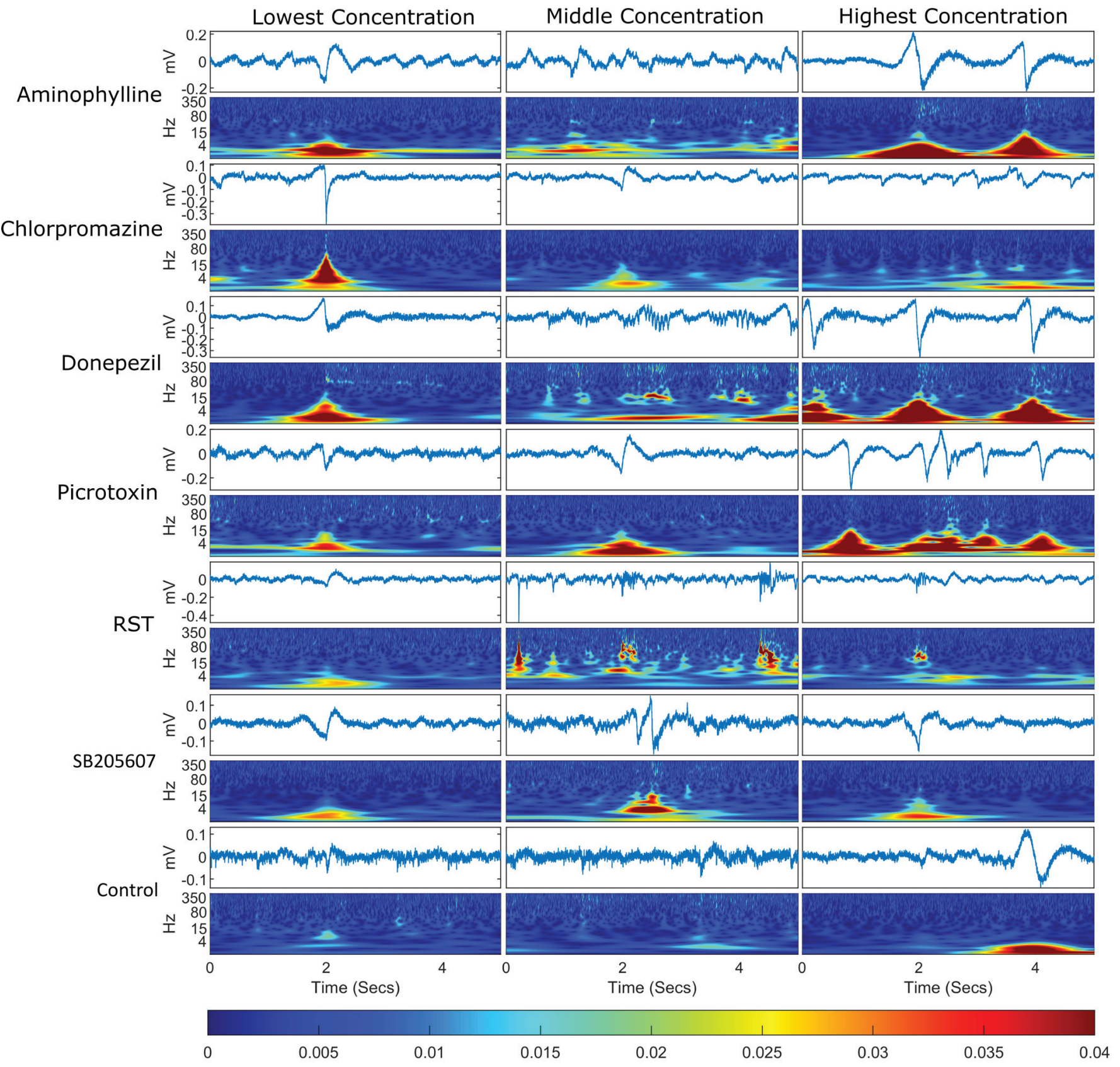




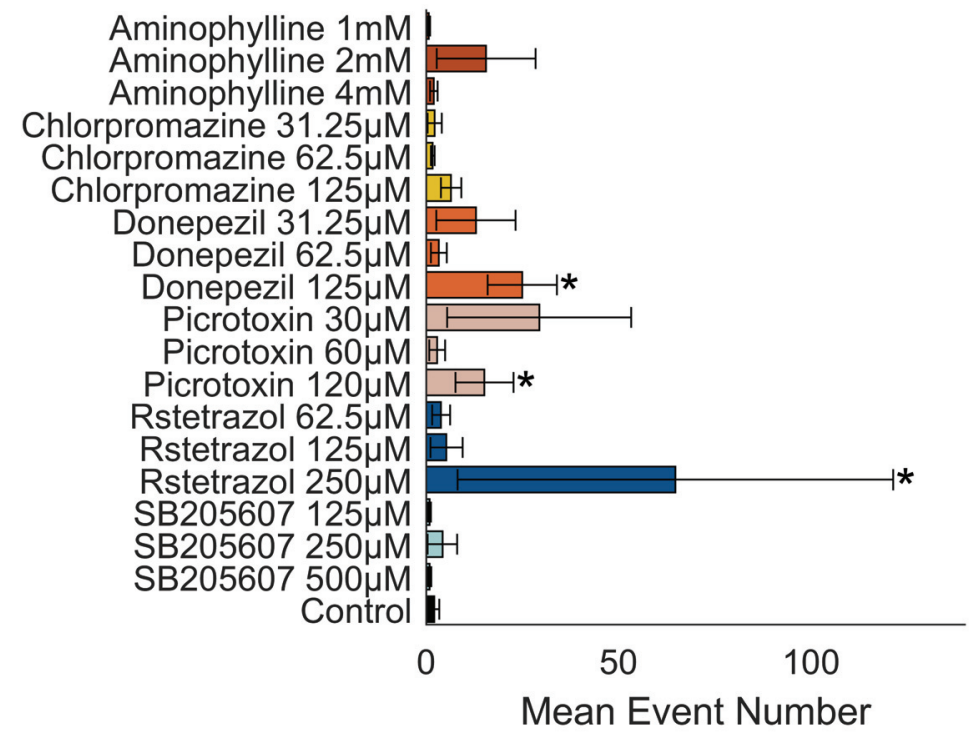



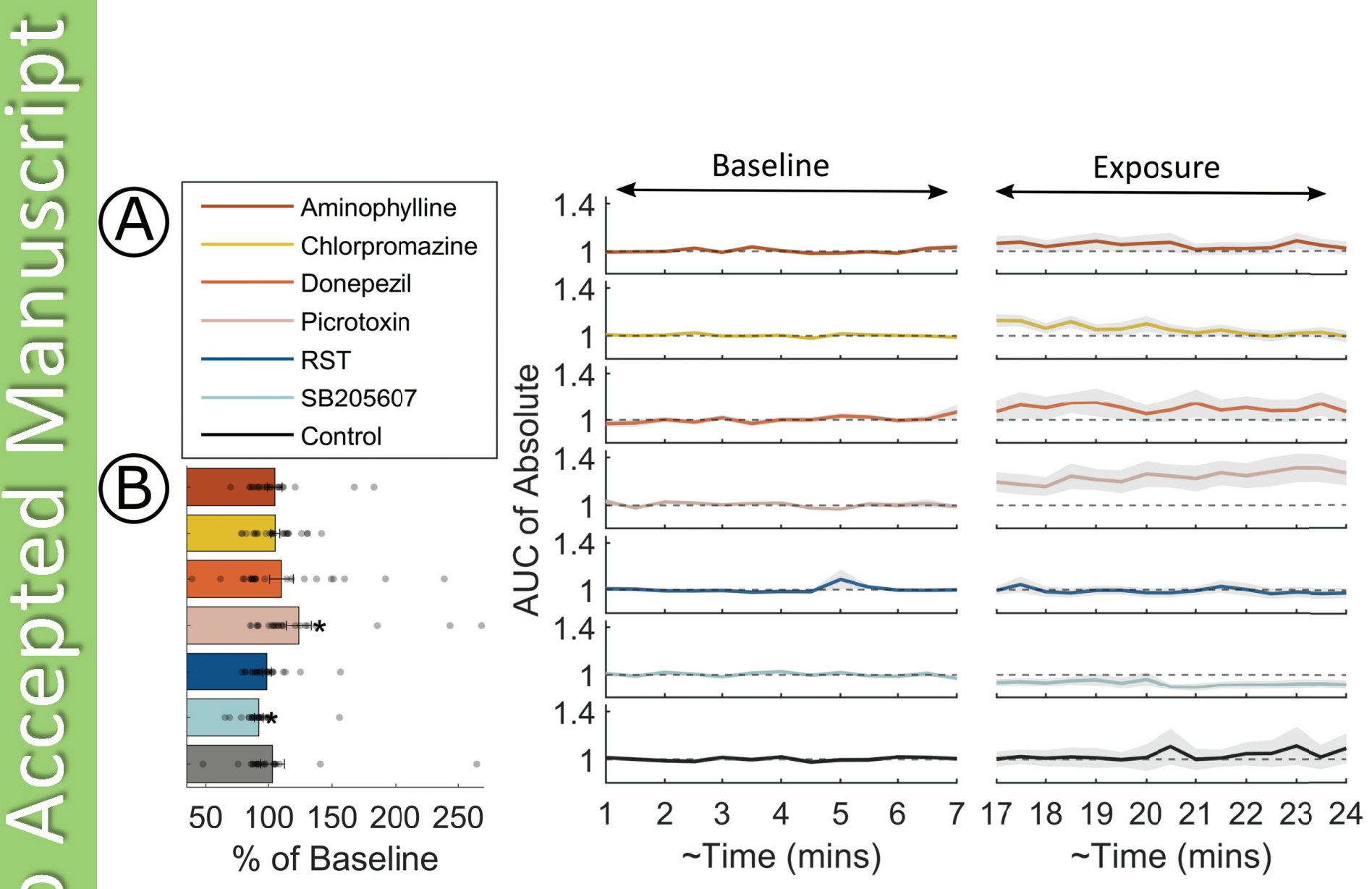
(A)

Aminophylline $1 \mathrm{mM}$ *

Aminophylline $2 \mathrm{mM}$ d*

Aminophylline $4 \mathrm{mM}$ *

Chlorpromazine $31.25 \mu \mathrm{M}$ **

Chlorpromazine $62.5 \mu \mathrm{M}$ *

Chlorpromazine $125 \mu \mathrm{M}$ ist

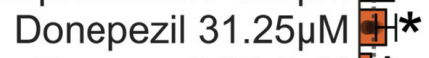

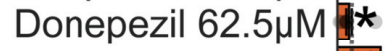

Donepezil $125 \mu \mathrm{M}$ *

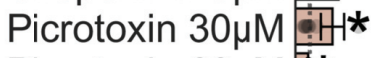

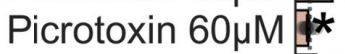

Picrotoxin $120 \mu \mathrm{M}$ ]*

Rstetrazol $62.5 \mu \mathrm{M}$ *

Rstetrazol $125 \mu \mathrm{M}$ *

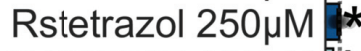

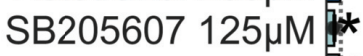

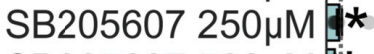

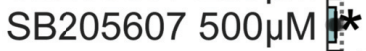

Control
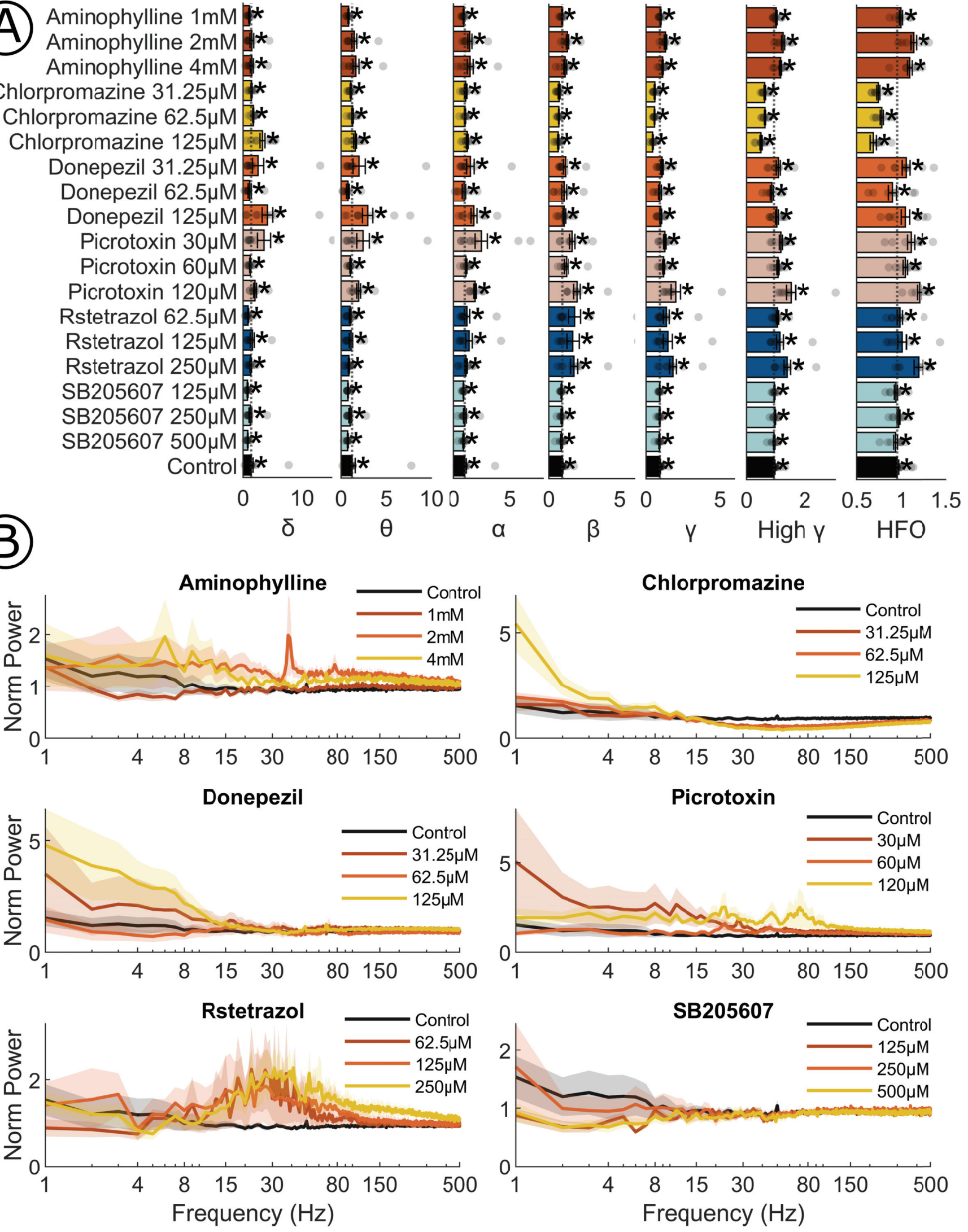

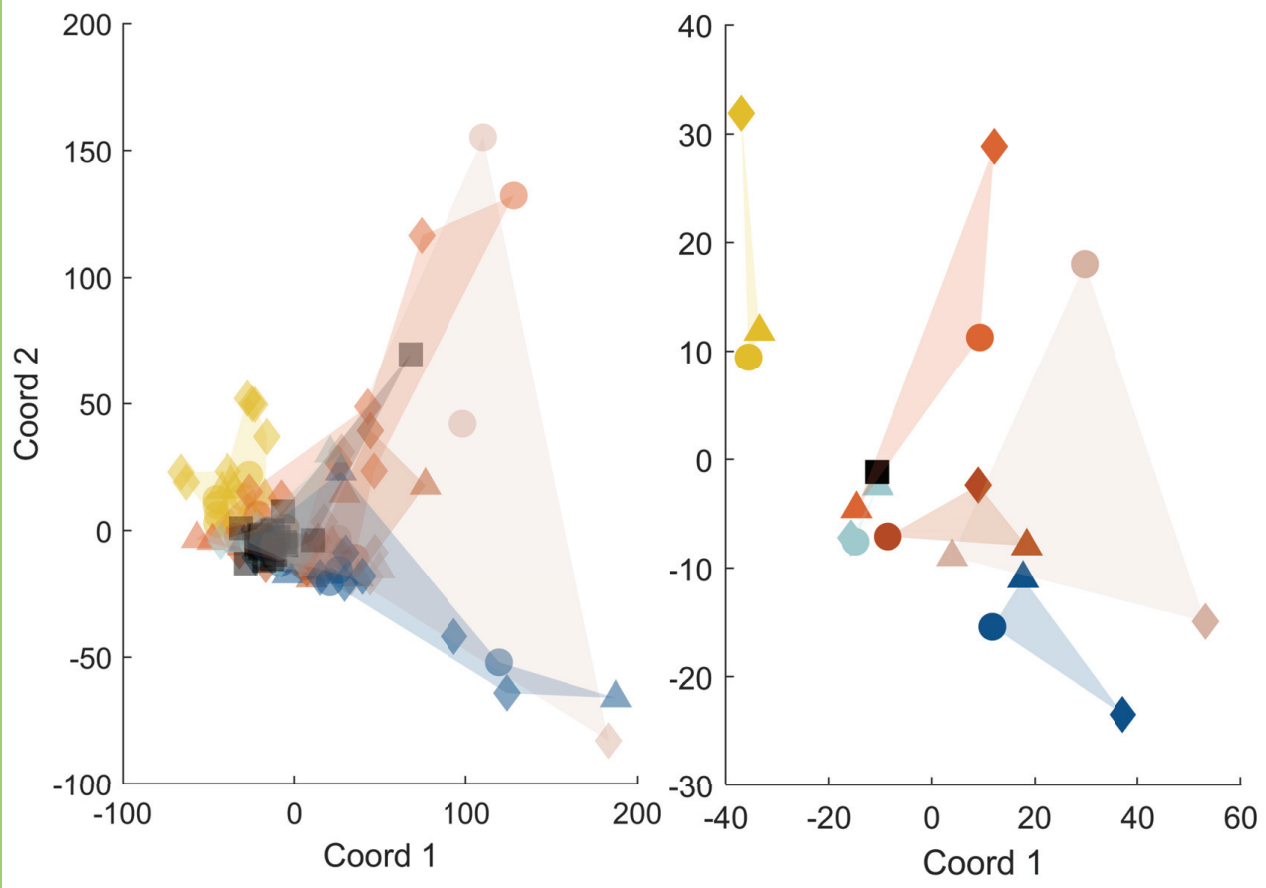

\begin{tabular}{|c|c|}
\hline$\bullet$ & Aminophylline $1 \mathrm{mM}$ \\
\hline$\Delta$ & Aminophylline $2 \mathrm{mM}$ \\
\hline$\diamond$ & Aminophylline $4 \mathrm{mM}$ \\
\hline$\bullet$ & Chlorpromazine $31.25 \mu \mathrm{M}$ \\
\hline$\Delta$ & Chlorpromazine $62.5 \mu \mathrm{M}$ \\
\hline$\diamond$ & Chlorpromazine $125 \mu \mathrm{M}$ \\
\hline$\bullet$ & Donepezil $31.25 \mu \mathrm{M}$ \\
\hline$\Delta$ & Donepezil $62.5 \mu \mathrm{M}$ \\
\hline$\diamond$ & Donepezil $125 \mu \mathrm{M}$ \\
\hline - & Picrotoxin $30 \mu \mathrm{M}$ \\
\hline$\Delta$ & Picrotoxin $60 \mu \mathrm{M}$ \\
\hline$\diamond$ & Picrotoxin $120 \mu \mathrm{M}$ \\
\hline - & Rstetrazol $62.5 \mu \mathrm{M}$ \\
\hline $\boldsymbol{\Delta}$ & Rstetrazol $125 \mu \mathrm{M}$ \\
\hline$\diamond$ & Rstetrazol $250 \mu \mathrm{M}$ \\
\hline - & SB205 $125 \mu \mathrm{M}$ \\
\hline$\Delta$ & SB205 $250 \mu \mathrm{M}$ \\
\hline 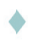 & SB205 $500 \mu \mathrm{M}$ \\
\hline - & Control \\
\hline
\end{tabular}

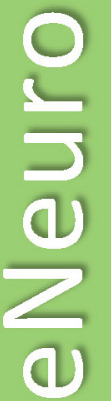

